\title{
Histone deacetylase inhibition sensitizes osteosarcoma to heavy ion radiotherapy
}

\author{
Claudia Blattmann ${ }^{2,4,5,7^{*}}$, Susanne Oertel ${ }^{1,4}$, Markus Thiemann ${ }^{2,4}$, Anne Dittmar ${ }^{1,4}$, Eva Roth ${ }^{2,4}$, Andreas E. Kulozik ${ }^{2,5,6}$, \\ Volker Ehemann ${ }^{3}$, Wilko Weichert ${ }^{3,5,6}$, Peter E. Huber ${ }^{4}$, Albrecht Stenzinger ${ }^{3}$ and Jürgen Debus ${ }^{1,4}$
}

\begin{abstract}
Background: Minimal improvements in treatment or survival of patients with osteosarcoma have been achieved during the last three decades. Especially in the case of incomplete tumor resection, prognosis remains poor. Heavy ion radiotherapy (HIT) and modern anticancer drugs like histone deacetylase inhibitors (HDACi) have shown promising effects in osteosarcoma in vitro. In this study, we tested the effect of HIT and the combination of HIT and the HDACi suberoylanilide hydroxamic acid (SAHA) in a xenograft mouse model.

Methods: Osteosarcoma xenografts were established by subcutaneous injection of KHOS-24OS cells and treated with either vehicle (DMSO), SAHA, HIT or HIT and SAHA. Tumor growth was determined and tumor necrosis, proliferation rate, apoptotic rate as well as vessel density were evaluated.

Results: Here, we show that the combination of HIT and SAHA induced a significant delay of tumor growth

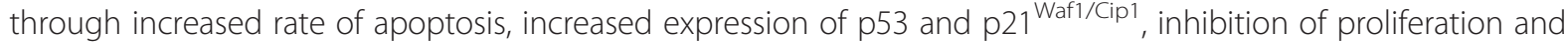
angiogenesis compared to tumors treated with HIT only.

Conclusion: HIT and in particular the combination of HIT and histone deacetylase inhibition is a promising treatment strategy in OS and may be tested in clinical trials.

Keywords: Osteosarcoma, Carbon ion radiotherapy, Histone deacetylase inhibition, Suberoylanilide hydroxamic acid, Radiosensitization, Mouse model
\end{abstract}

\section{Background}

Osteosarcoma (OS) is the most frequent primary malignant bone tumor in children and adolescents. They account for up to $15 \%$ of all extracranial solid neoplasms in patients aged 15-19 years. Despite significant advancements in the diagnosis and treatment to date, overall survival has remained relatively constant for about three decades and is still poor for non-resectable tumors and advanced metastatic disease [1]. Until now the only therapy that is validated in a large number of patients is complete tumor resection combined with neo-adjuvant and adjuvant chemotherapy to establish local control. With this therapy regimen, the survival rate for patients with localized osteosarcoma is in the range of $60-70 \%$,

\footnotetext{
* Correspondence: c.blattmann@klinikum-stuttgart.de

${ }^{2}$ Department of Pediatric Oncology, Hematology and Immunology, University Children's, Hospital of Heidelberg, Heidelberg, Germany ${ }^{4}$ Department of Radiation Oncology, German Cancer Research Center, Heidelberg, Germany

Full list of author information is available at the end of the article
}

for patients with metastases in the range of 15-30\%. However, there is also a considerable fraction of patients (about $10 \%$ ) with non-resectable tumors. Moreover surgery is often denied by patients when a complete surgical resection can only be reached by amputation of a limb. In these cases radiation therapy and chemotherapy is a possible option that has been validated in a limited number of patients [2]. Radiotherapy in this setting is still being discussed controversially: particularly for children since high radiation doses are required, bearing unwanted side-effects including acute and late local toxicity and the considerable risk for secondary malignancies. Therefore novel therapeutic approaches like irradiation with heavy ions (HIT) or the use of radiation sensitizers that selectively augment the response of osteosarcomas to radiation and thus increase the therapeutic ratio are urgently needed.

HIT offers significant advantages in comparison to conventional photon therapy (XRT). The physical depth- 
dose distribution in tissue of heavy ions is characterized by a small entrance dose and a distinct maximum (Bragg peak) near the end of range with a sharp fall-off at the distal edge. These facts allow delivery of the dose with millimeter precision. In addition, HIT exhibit an enhanced biological effectiveness in the Bragg peak region caused by the dense ionization of individual particle tracks resulting in reduced cellular repair. These advantages will potentially reduce undesirable side-effects and the risk of secondary malignancies as well as improve outcome and quality of life. Therefore, it makes them particularly attractive for the treatment of pediatric patients and young adults as well as for radio-resistant tumors such as osteosarcomas [3-6].

Histone deacetylase inhibitiors (HDACi) are epigenetic modulators with manifold effects on tumor cells [7]. In a number of in vitro and in vivo experimental models, HDACi have been shown to act as radiosensitizers in a number of solide tumors including gliomas, colorectal carcinomas and melanoma [8-11]. The HDACi suberoylanilide hydroxamic acid (SAHA) has been approved for the treatment of T-cell lymphoma. Furthermore, promising effects of SAHA in solide tumors have been shown clinical trials [12]. Recently, we showed a significant radiosensitizing effect of SAHA in combination with $\mathrm{XRT}$ in osteosarcoma in vivo [13]. First in vitro data showed promising effects by the combination HIT and SAHA in infantile sarcoma cell lines $[14,15]$.

The aim of the present study was to evaluate the efficacy of HIT alone and in combination with SAHA as well as potential underlying mechanisms in osteosarcomas in vivo.

\section{Methods}

\section{Cells and reagents}

The KHOS-24OS human OS cell line was purchased from the American Type Culture Collection (ATCC; Rockville, $\mathrm{MD)}$ and maintained in complete culture medium (DMEM) supplemented with 10 \% FCS. SAHA was obtained from Alexis Biochemicals (Lörrach, Germany), DMSO from Carl Roth Biochemicals (Karlsruhe, Germany). Ketamine (0.4 mg/20 g BW) and xylazine (Bayer Germany) (90 mg/20 g BW) were used to anesthetize mice during radiation treatments.

\section{Animal model}

All animal experiments were approved by the ethics committee of the "Regierungspräsidium Karlsruhe" (reference number 35-9185.81/G-165/08).

For the pilot experiment were 30 mice used to define the HIT dose: control group and irradiated mice with 2 Gy, each with 5 mice, irradiated mice with $1,3,5,10$ and $20 \mathrm{~Gy}$, each with 4 mice.
The number of mice for the main experiment was calculated with 24 mice per treatment group. Xenografts of human KHOS-24OS cells were established by subcutaneous inoculation of tumor fragments (diameter 1-2 mm) into the hind legs of 10 weeks old SCID mice (CB17/Icr$P r k d c^{s c i d} / \mathrm{IcrCrl}$ ) (Charles River, Wilmington, Mass.). The tumor fragments were generated from a donor animal after inoculation of $5 \times 10^{6}$ KHOS-24OS cells into the hind legs. When the tumor reached a size of about $14 \times \mathrm{e} 14 \mathrm{~mm}$, they were fragmented and implanted into all experimental animals. In a preliminary dose finding trial 6 different doses between 1 and 20 Gy of carbon ions were given and compared to a control group. Every group consisted of 5 mice. The mice were maintained under specific pathogen-free conditions, food and water were supplied ad libidum. Housing and all procedures involving the mice were performed according to the protocols approved by the local regional board.

When tumors reached $90-100 \mathrm{~mm}^{3}$, mice were randomly assigned to one of four treatment groups: vehicle (DMSO) control (24 animals), SAHA alone (21 animals), radiation alone (20 animals), or combined SAHA and radiation (21 animals). In total, 10 of 96 animals died during the investigation period due to anesthesia-related complications ( 3 mice in the SAHA group, 4 mice in the XRT group and 3 mice in the combination treatment group. Therefore, these groups are smaller than the control group. SAHA $100 \mathrm{mg} / \mathrm{kg}$ was solubilized in DMSO $(99.5 \%)$, given intraperitoneally (i.p.), started $24 \mathrm{~h}$ before radiation and applied for 5 days a week, 3 weeks in total. On day 1 , mice tumors were irradiated with carbon ions in a single dose of 2.5 Gy. For HIT, mice were anesthetized with ketamine and xylazine and then exposed to HIT directed at the tumor site. In the following, the mice were weighed, and the tumor sizes were measured using a caliper twice a week. Tumor length (L) and width (W) were measured and tumor volume calculated as $\left(\mathrm{L} \times \mathrm{W}^{2} / 2\right)$, where $\mathrm{L}=$ longest diameter and $\mathrm{W}=$ shortest diameter. For each tumor, tumor size and multiplication to initial tumor size was assessed and documented. Animals were euthanized by cervical dislocation when tumors reached a volume of $2.0 \mathrm{~cm}^{3}$ or severe necrosis latest 45 days after treatment start.

\section{Immunohistochemical analysis}

Immunohistochemistry was performed in three tumors per treatment group at the following time points: $24 \mathrm{~h}$, 8 days, 24 and 45 days after HIT.

3 micrometre thick whole tumor sections were cut from formalin-fixed, paraffin-embedded (FFPE) tissue blocks. Sections were stained with hematoxylin-eosin (HE; SigmaAldrich, St Louis, USA). On these H\&E stained slides, the total amount of necrotic versus vital tissue was analyzed 
in $5 \%$ increments and means for all treatment groups were calculated.

Immunohistochemical staining for the evaluation of proliferative capacity and microvessel density was carried out with anti- Ki-67 (monoclonal mouse clone MIB-1, 1:200; Dako, Hamburg, Germany) and anti- CD34 (1:25, pH6; Dako, Hamburg, Germany) antibodies as recommended by the manufacturer.

Ki-67-positive tumor cell nuclei were counted in 5 representative areas of each tumor. Evaluation of vessel density was done on the slides stained for CD34. The two most vascularized areas within a given tumor ('hot spots') were chosen at low magnification $(\times 10)$ and vessels were counted in a representative high magnification $(\times 400$; image size $385.9 \times 251 \mu \mathrm{m}$ ) field in each of these two areas. Single immunoreactive endothelial cells, or endothelial cell clusters separate from other microvessels, were counted as individual microvessels. Endothelial staining in large vessels with tunica media, and nonspecific staining of nonendothelial structures, was disregarded.

Apoptosis was detected by an in situ apoptosis detection kit, ApopTAG ${ }^{\circ}$ (S7100; Chemicon International (Millipore), Temecula, CA, USA) on $3 \mu \mathrm{m}$ thick formalin-fixed, paraffin-embedded whole tumor sections as specified by the manufacturer. TUNEL-positive cells were counted at 40x magnification 5 representative areas of each tumor.

For protein detection, the following primary antibodies were employed: rabbit monoclonal anti-p21 (1:100, pH9, Abcam, Cambridge, UK; \#ab92675) and anti-p53 (1:100, pH9, Dako, Hamburg, Germany. After heat-induced antigen retrieval, slides were incubated with primary antibody at $4{ }^{\circ} \mathrm{C}$ overnight. Bound antibody was detected by a Super Sensitive IHC-Detection- System (BioGenex, San Ramon, CA). For colour development, a DAB system (DAKO, Hamburg, Germany) was used. The scores of $\mathrm{p} 21^{\text {Waf1/Cip } 1}$ and $\mathrm{p} 53$ expression were calculated by multiplication of staining intensity and staining percentage. Staining intensity was defined as follows: $0=$ no staining, 1 = weak staining, $2=$ moderate and $3=$ strong staining. Staining percentage was defined as follows: $0=$ no cells, $1=1-10 \%$ cells, $2=11-50 \%$ cells, $3=51-$ $80 \%$ and $4>80 \%$ cells. Evaluation of all tissue based parameters was done by two experienced pathologist (WW and AS) together on a multiheaded microscope. Both observers were blinded to the mouse treatment allocation.

\section{Data analysis}

The two-sided $t$-test was used to analyze the differences between the treatment groups. $P$ values $<0.05$ were considered statistically significant. Data are presented either as mean or as median, error estimation was performed by calculation of the standard error of mean (SEM).
Actual tumor growth delay was calculated with (T'x-Tx)/ Tx as the time it took the irradiated tumors ( $\left.\mathrm{T}^{\prime}\right)$ and the tumors of the control group to $\mathrm{x}$-fold multiply their volume. Local control was estimated by Kaplan-Meier curves, and the differences in time-to-failure (TTF) between groups were assessed using the log-rank test.

\section{Ethical statement}

All authors confirm that the animal experiments comply with the "Animal Research: Reporting In Vivo Experiments" (ARRIVE) guidelines.

\section{Results}

Previously, we assessed the potency of XRT and SAHA in osteosarcoma xenografts [13]. Now, HIT and SAHA were investigated using the same mouse model and results were compared.

\section{HIT is superior to XRT in OS xenografts}

Our dosimetry trial revealed that a higher biologic effectiveness of HIT compared to XRT in our osteosarcoma model can be observed especially at higher single dose levels above 5 Gy (Fig. 1). On the basis of these data, for the combination trial a single dose of 2.5 Gy was picked in order to prevent from covering up a possible supraadditive effect. This dose showed significant growth retardation within the first 14 days after treatment, comparable to that observed after 5 Gy with XRT, but was not too effective to mask the anticipated additional effect of SAHA (Fig. 2).

\section{SAHA sensitizes OS to HIT in vivo}

Mice bearing KHOS-24OS xenografts were injected i.p. with SAHA (100 mg/kg) for 5 days a week, 15 days in total, starting $24 \mathrm{~h}$ before HIT with a dosage of $2.5 \mathrm{~Gy}$.

SAHA given as a single-agent for 15 days induced no significant tumor growth delay compared to mice of the control group.

In analogy to our recent publication about the combination of XRT with SAHA [16], the combination of HIT with SAHA led to a significantly delayed tumor growth rate compared to HIT alone $\left(\mathrm{p}_{\mathrm{d} 23}=0.005\right)$. Comparing HIT as mono-treatment to SAHA only, HIT seemed to be superior from day 10 on after treatment start reaching significance at day $24\left(\mathrm{p}_{\mathrm{d} 24}=0.04\right)$. The combination of HIT and SAHA yielded a significant tumor growth retardation (measured by tumor volume) compared to SAHA only and HIT only starting day $20\left(\mathrm{p}_{\mathrm{d} 20}=0.005\right)$ and day 25 $\left(\mathrm{p}_{\mathrm{d} 25}=0.04\right)$ respectively (Fig. 3$)$.

The significant $(p<0,05)$ superior effect of HIT and SAHA was most pronounced between days 25 and 35 after treatment as visualized after calculation according to Kaplan-Meier. As planned, neither treatment was 


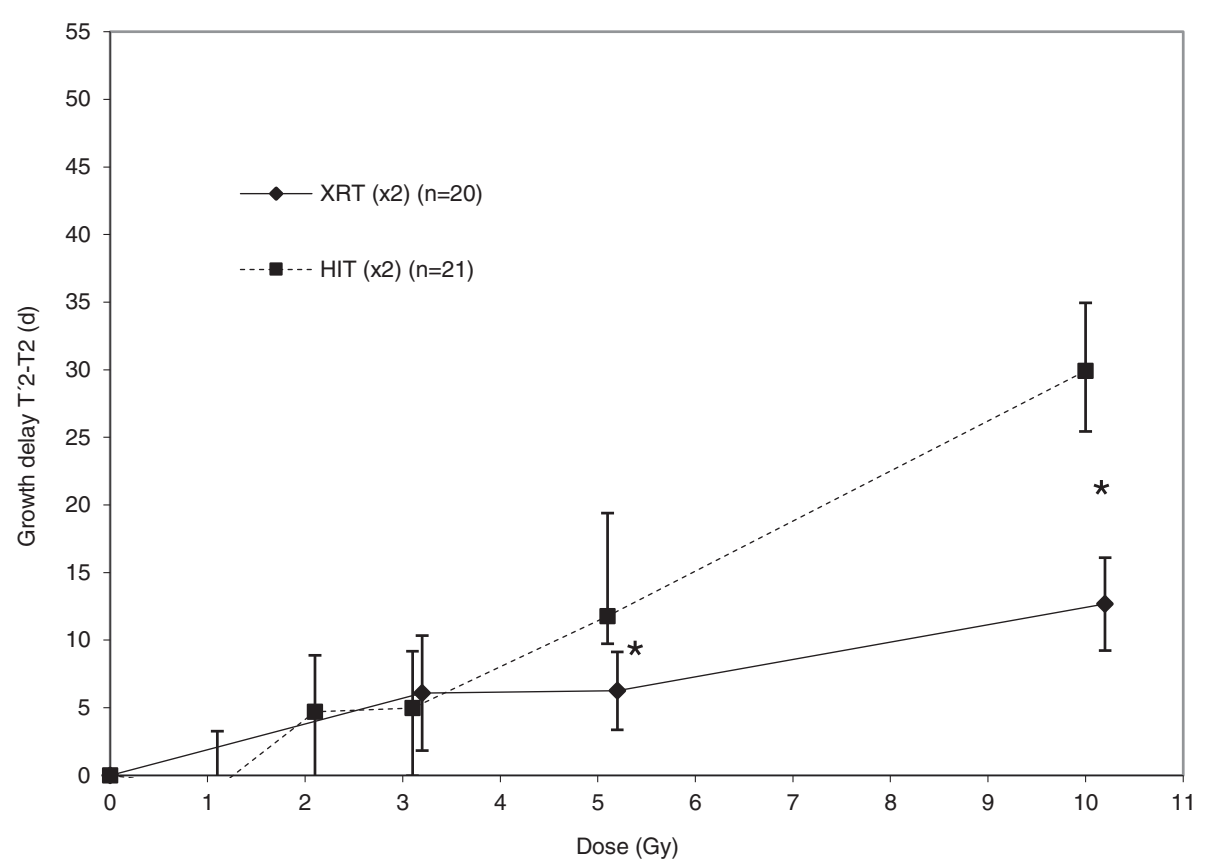

Fig. 1 HIT is superior to XRT in osteosarcoma xenografts. Tumor duplication after treatment with different doses of HIT and XRT was compared in to animal groups. Actual tumor growth delay was calculated with $\left(T^{\prime} x-T x\right) / T x$ as the time taken for the irradiated tumors $\left(T^{\prime}\right)$ and the control tumors $(T)$ to $x$-fold multiply their volume $(x)$

dosed intensively enough to yield tumor control beyond 40 days (Fig. 4).

Heavy ion radiation and SAHA application were tolerated without observable toxicity in mice. In total, 4 of 86 animals died during the investigation period due to anesthesia-related complications. After treatment all mice were weighed once a week. Neither variation in mean weight between the four groups nor severe weight loss in any mice were observable.

Dose enhancement factor at triplication of tumor volume was 1.7 in the SAHA plus HIT treated group, 1.4 in the HIT treated group and 1.1 and in the SAHA treated group.

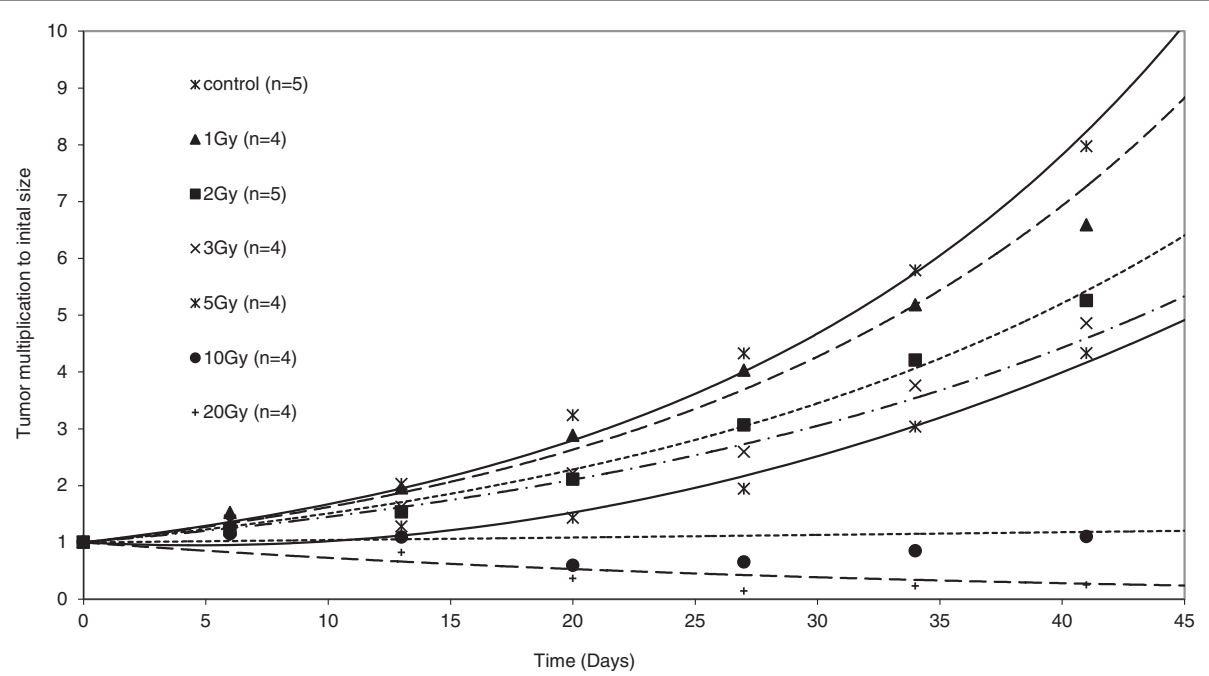

Fig. 2 The HIT dosage of 2.5 Gy leads to a tumor growth delay of 14 days. In a preliminary test, xenografts were irradiated with a single dose of $1,2,3,5,10$ or $20 \mathrm{~Gy}$. The dose of $2.5 \mathrm{~Gy}$ were chosen for our further experiments because significant but not to effective growth retardation within the first 14 days after treatment was seen leaving capacity for the anticipated additional effect of SAHA 


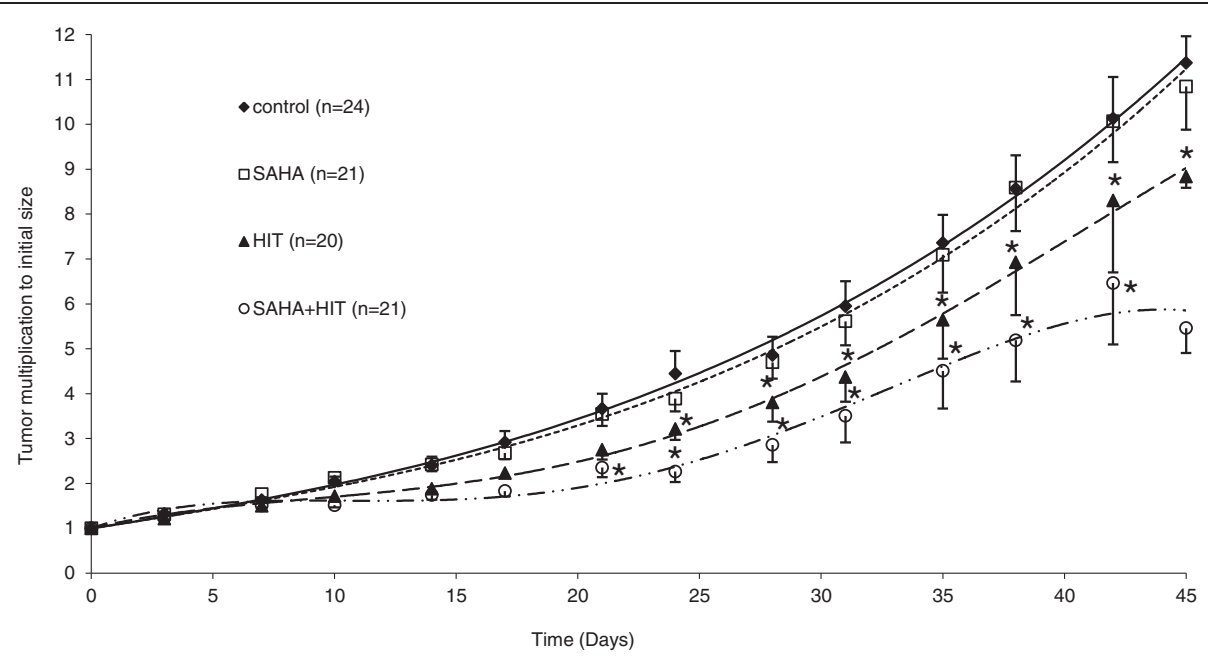

Fig. 3 The combination of HIT and SAHA results in a significant tumor growth delay compared to treatment with HIT or SAHA only. Osteosarcoma xenografts were treated with DMSO (controls), suberoylanilide hydroxamic acid (SAHA), irradiation (HIT) or SAHA plus HIT and tumor growth was determined until day 45 after HIT. Comparing HIT as mono-treatment to SAHA only, HIT seemed to be superior from day 10 on after treatment start reaching significance at day 23. The combination of HIT and SAHA yielded a significant $\left(^{*}\right)$ tumor growth retardation compared to SAHA only and HIT only starting day 20 and day 25 respectively

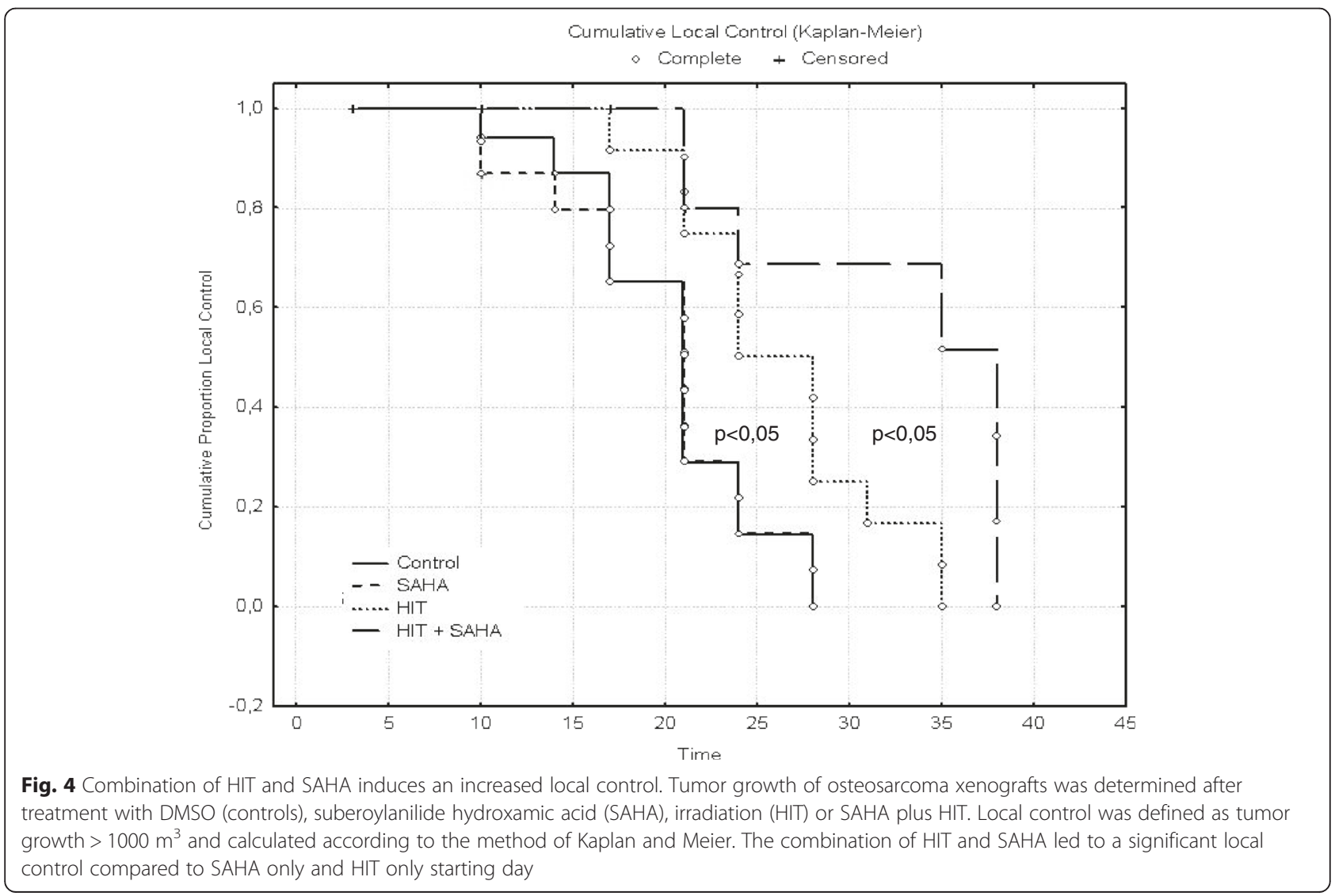


The combination of HIT and SAHA inhibits proliferation

Analysis of tumors was performed $24 \mathrm{~h}$, as well as 8, 24 and 45 days after HIT.

The proliferation rate was significantly reduced in tumors after treatment with SAHA and HIT at all investigation time points. HIT only treatment led to a significantly lower proliferation rate 8 and 24 days after irradiation compared to the control group. SAHA only treatment had no significant effect on tumor proliferation (Fig. 5).

\section{HIT and SAHA treatment induces apoptosis and necrosis}

The TUNEL assay showed a significant induction of apoptosis after treatment with HIT and SAHA compared to HIT only treatment 1,8 and 45 days $(p=0.002)$ after

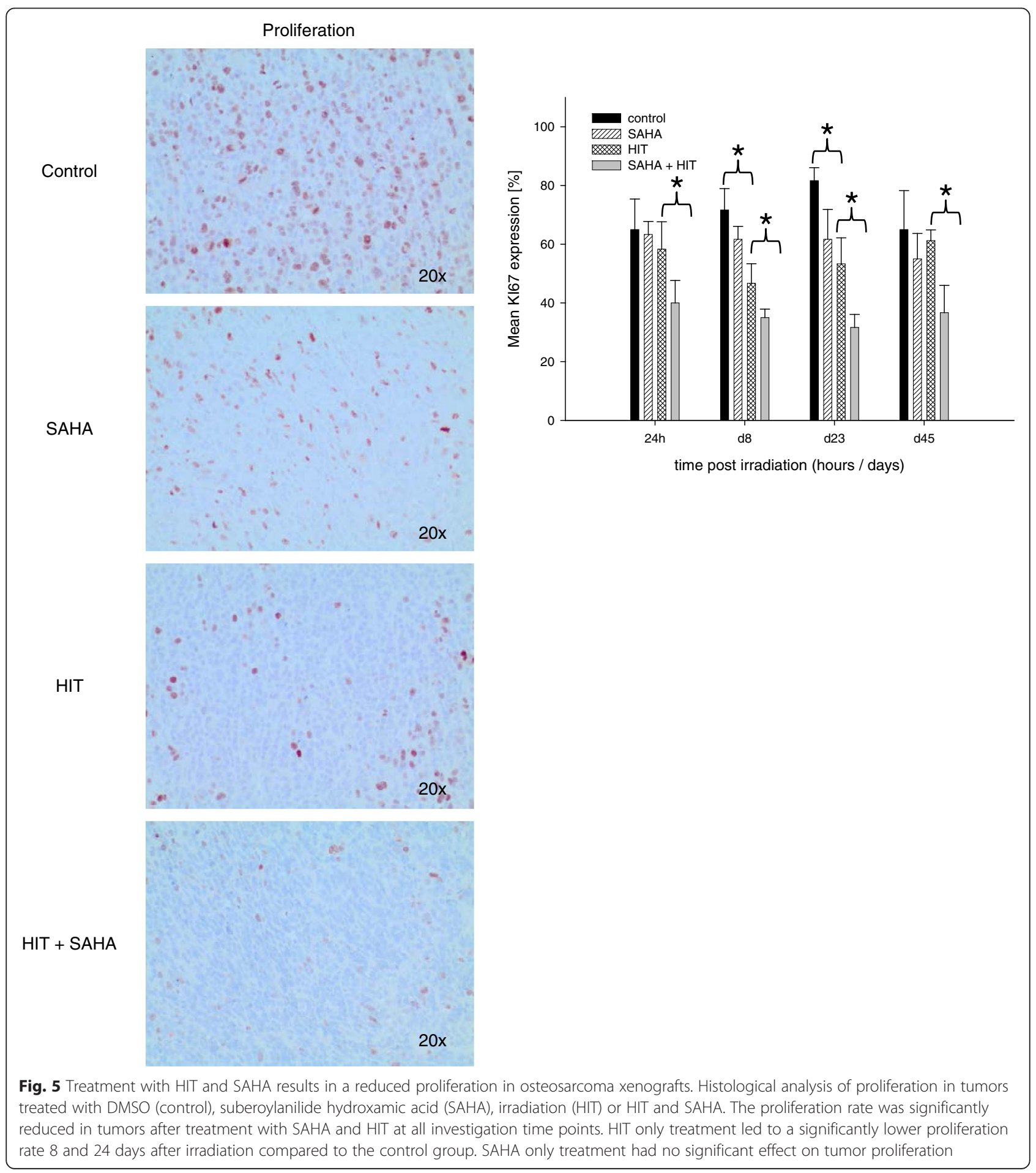


HIT. Apoptosis was also increased after SAHA only and HIT only 24 h, 8 and 45 days after irradiation but not as much as treatment with HIT and SAHA (Fig. 6).

We further analyzed expression of p53 and p21 Waf1/Cip1 in three tumors per treatment group. Our results revealed a higher p53 expression in all SAHA treated tumors compared to the vehicle treated control and only irradiated tumors on day 24 and 45 . p $21^{\text {Waf1/Cip } 1}$ expression was increased in tumors after SAHA only and SAHA and HIT treatment compared to the controls and the HIT treated tumors on day 8 but not at other points of time (Fig. 7).

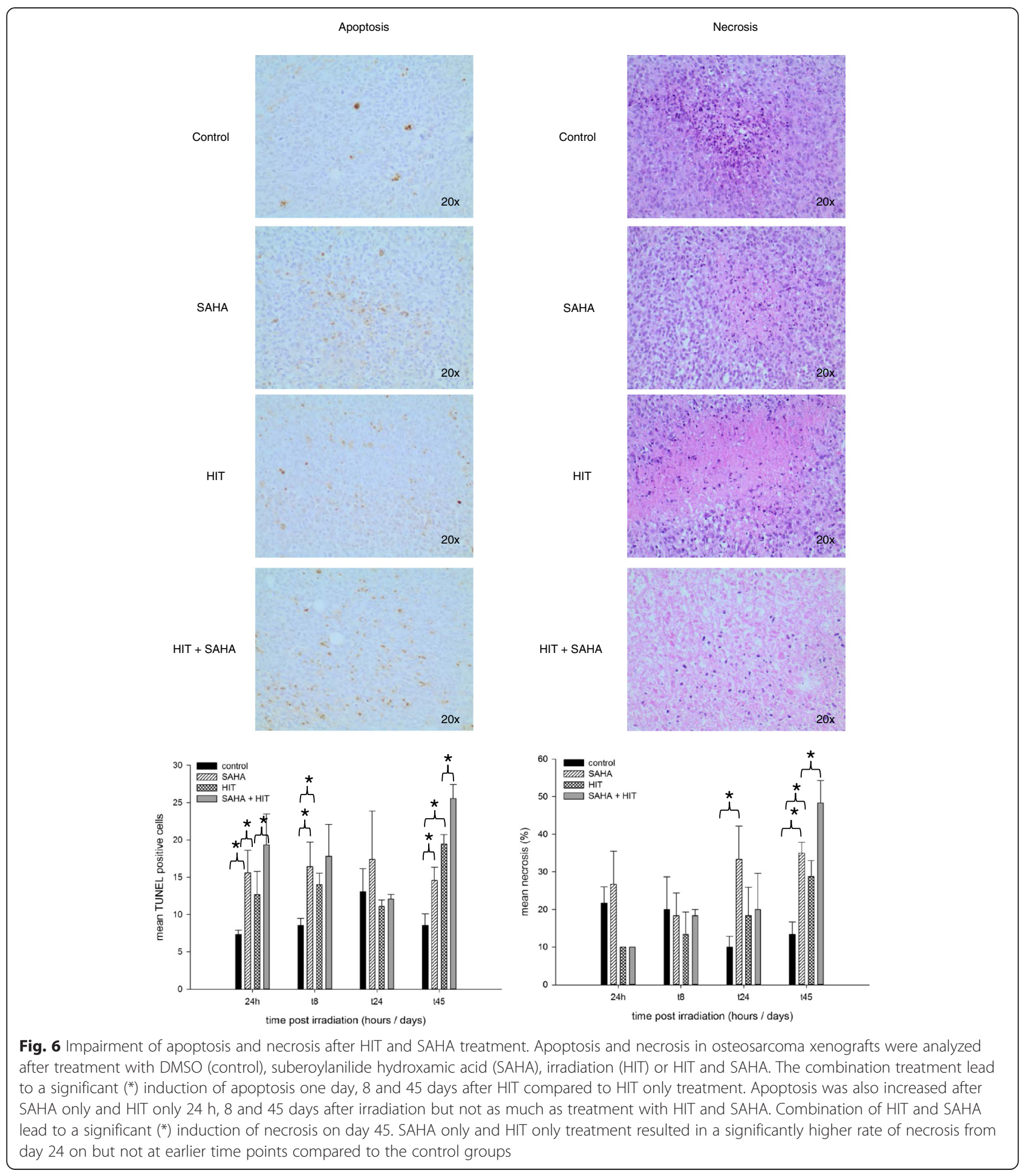


p53
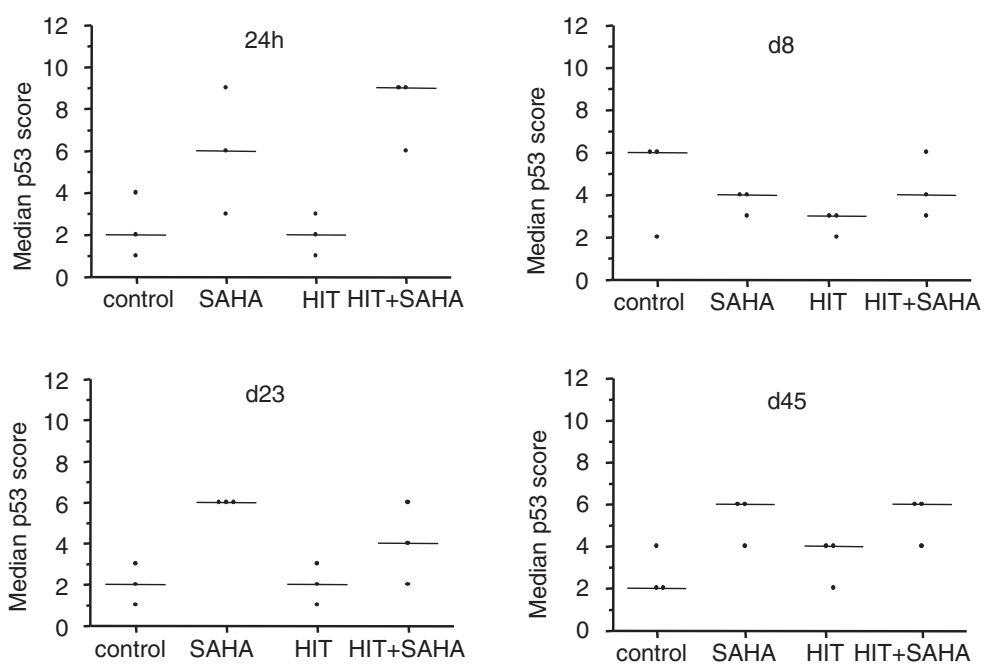

p21
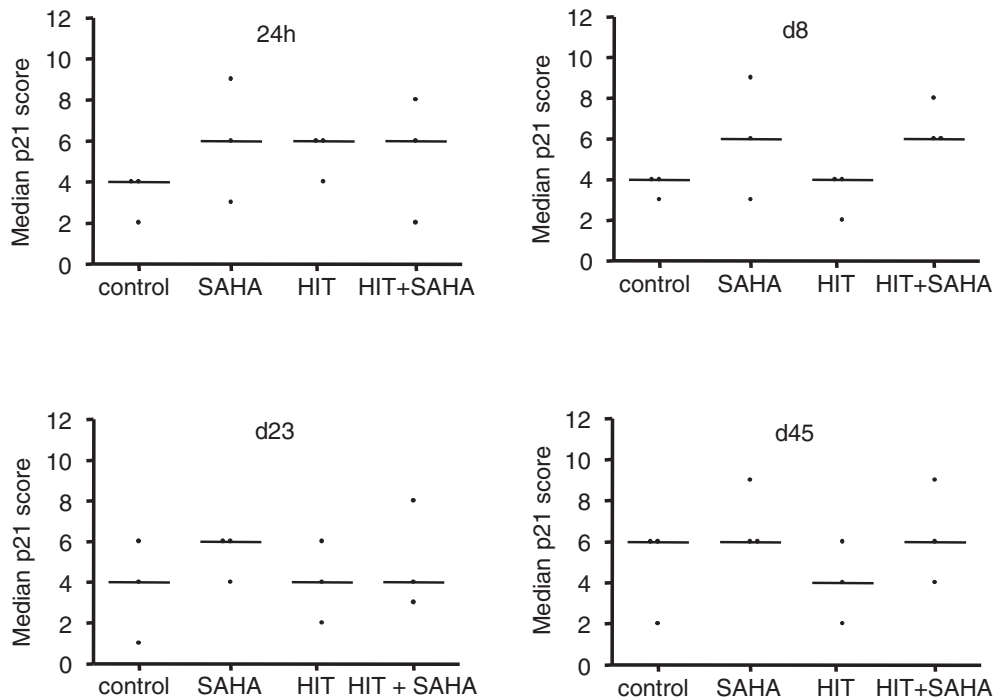

Fig. 7 Expression of $p 53$ and $p 21^{\text {WAF1/CIP1 }}$ is impaired in osteosarcoma xenografts after treatment with HIT and SAHA. Expression of p53 and p21 ${ }^{\text {WAF1/CIP1 }}$ was analyzed after treatment with DMSO (control), suberoylanilide hydroxamic acid (SAHA), irradiation (HIT) or HIT and SAHA. p53 expression was increased in all SAHA treated tumors compared to the vehicle treated control and only irradiated tumors on day 23 and 45. Increased p21 Waf1/Cip1 expression was detected in tumors after SAHA only and SAHA and HIT treatment compared to the controls and the HIT treated tumors on day 8

After treatment with SAHA only, the rate of necrosis was significantly increased on day 45 compared to the controls but not before (Fig. 7).

Combination of HIT and SAHA lead to a significant induction of necrosis on day $45(p=0.01)$. Furthermore, SAHA only and HIT only treatment resulted in a significantly higher rate of necrosis from day 24 on but not at earlier time points compared to the control groups (Fig. 6). However, rate of necrosis was highest in the combination treatment group.

\section{SAHA significantly impairs angiogenesis in combination} with HIT

We also evaluated the number of microvessels by CD34 immunohistochemistry. Compared to the control group, the density of microvessels was reduced in tumors of all treatment groups at all investigation time points. Tumors treated with HIT and SAHA showed a significant lower vascularization compared to tumors treated with HIT from day 24 on $(p=0.02)$ and the lowest vascularization at all (Fig. 8). 


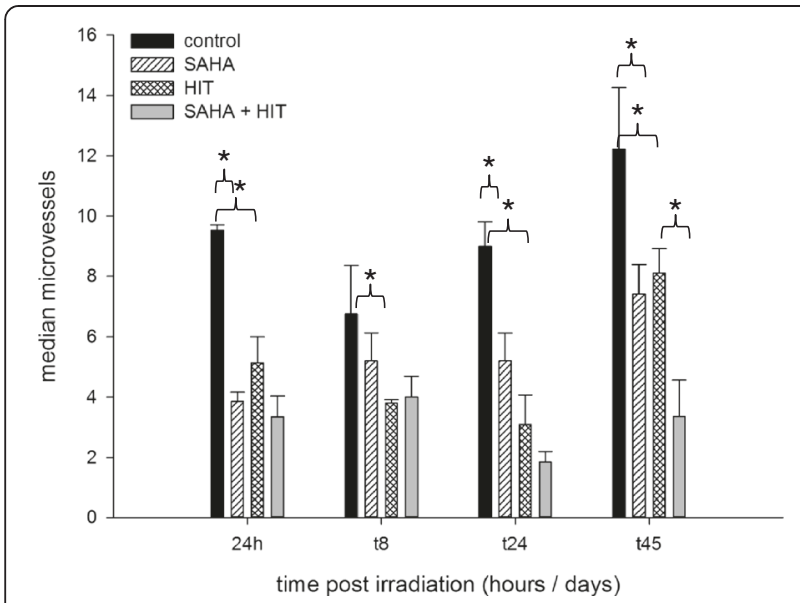

Fig. 8 Density of microvessels is significantly reduced in osteosarcoma after combination treatment. Xenografts were treated with DMSO (control), suberoylanilide hydroxamic acid (SAHA), irradiation (HIT) or HIT and SAHA. The density of microvessels was reduced in tumors of all treatment groups at all investigation time points. Tumors treated with HIT and SAHA showed a significant (*) lower vascularization compared to tumors treated with HIT from day 24 on and the lowest vascularization at all

\section{Discussion}

Osteosarcoma are highly malignant bone tumors and rather resistant to radiotherapy. Prognosis is fatal whenever local control cannot be achieved by surgery or/and radiotherapy $[17,18]$. Therefore, for patients with inoperable or not completely resectable osteosarcoma new treatment strategies with novel irradiation techniques like proton or heavy ion beams or new therapeutic substances with radiosensitizing effects are under investigation. HDACi have been shown to be effective anticancer agents [12]. In solid tumors, the full therapeutic potential of HDACi can, however, only be achieved in combination with other agents and / or radiation [19,20]. SAHA is one of the well-established HDACi and has been shown to act as a radiosensitizer in vitro and in vivo in different tumor entities [7-10,12,13]. The current study is the first that investigates the efficacy of HDACi in combination with HIT, the most effective form of radiation in osteosarcoma in clinical practice [21].

Comparing the current study with the results of our recently published study [12], we observed a higher efficacy of HIT at higher single doses $>5$ Gy. Although these data correspond well to previous findings, this observation needs to be reevaluated as the rate of growth in the control animals of the two subsequently performed studies varied considerably.

Furthermore, our study clearly shows that SAHA significantly and effectively radiosensitizes osteosarcoma to HIT in vivo and that the combination of SAHA and HIT is superior to either agent alone. Growth of tumors treated with SAHA and HIT was significantly delayed compared to tumors treated with either HIT only or SAHA without observable toxicity in our mice.

Immunhistochemical analysis of tumor xenografts revealed a significant increase of necrosis on day 45 after HIT and SAHA treatment compared to HIT alone. Proliferation was impaired in tumors after combination treatment compared to tumors treated with HIT only. Furthermore, apoptosis was significantly induced by treatment with HIT and SAHA compared to irradiated tumors.

Our results are in line with the observations of many other studies [22-25]. HDACi are regarded as agents capable of reactivating apoptosis in tumor cells by affecting very different parts of the apoptosis cascade; for example, through increased expression of death receptors like tumor necrosis factor-related apoptosis-inducing ligand (TRAIL) cell surface receptors or by directly stimulating a death receptor pathway without altered receptor expression [26]. Henderson et al. reported about HDACi - induced cell death by activation of caspases but also could show that inhibition of caspases did not block HDACi induced cell death [27]. Furthermore, apoptosis-inducing effects of HDACi might not only be due to histone deacetylation and subsequent transcriptional regulation. $\mathrm{HDACi}$ can also block deacetylation of important proteins, such as the tumor suppressor gene TP53 [28]. P53 is one of the most commonly altered transcription factors in cancer and plays a pivotal role in the cellular response to DNAdamaging agents [29]. A number of studies demonstrate activation of p53 in cells after exposure to HDACi and ability of HDACi to enhance radiation response in cancer cells through increase of p53 acetylation-phosphorylation [30-32]. Correspondingly, we were able to observe an increase of p53 expression in all SAHA treated samples. However, there was no difference between SAHA only and SAHA plus HIT treated tumors and no supraadditive effect that might prove a sensitization to HIT beyond the SAHA only effect.

HDACi-mediated effects on the cell cycle are also postulated to be a key reason for their toxicity in tumor cells. Most HDACi lead to a cell cycle arrest at G1 associated with induction of CDKN1A/p21 WAF1/CIP1 [33]. Recently, we observed a HDACi-induced cell cycle arrest at G1 accompanied with up-regulation of $\mathrm{p} 21^{\mathrm{WAF} 1 / \mathrm{CIP} 1}$ in vitro. However, this effect does not fully explain the radiosensitizing property because multiple underlying mechanisms are described like effects on DNA damage repair, angiogenesis and apoptosis.

Solid tumors are frequently angiogenesis dependent. The immunohistochemical assessment of angiogenesis in our animal model showed a significant decrease of microvessels in all tumors treated with HIT and SAHA compared to tumors treated with either HIT or SAHA. Vascularization of malignant lesions depends on the 
expression of specific genes in both endothelial and tumor cells. There is evidence that several members of the histone deacetylase family play key roles in the regulation of these genes. Indeed, numerous in vitro and in vivo studies demonstrated that inhibitors of HDAC modulate angiogenic gene expression in both endothelial and cancer cells and disturb the delicate and complex balance between the collective action of pro-angiogenic factors and angiogenesis inhibitors [34].

\section{Conclusions}

Taken together, we demonstrate that SAHA leads to a significant tumor growth delay of OS in vivo when combined with HIT. This effect in our in vivo model is most likely caused by a combination of an induction of apoptosis, an impairment of angiogenesis and a supraadditive impact of the combination treatment on proliferation. Our results indicate that a combination of HDACi and HIT might be a strategy therapeutic option for patients with OS, particularly if tumors are non-resectable.

\section{Abbreviations \\ HDACi: Histone deacetylase inhibitor; HIT: Heavy ions; SAHA: Suberoylanilide hydroxamic acid; XRT: Conventional photon therapy.}

\section{Competing interests}

The authors declare that they have no competing interests.

\section{Authors' contributions}

BC and OS conceived the study, wrote the application to the ethic committee, participated in the animal and cell line experiments and drafted the manuscript. TM developed and carried out the animal experiments. REK and DA participated in the animal and cell line experiments. SA, EV and WW carried out the histological examinations. HPE participated in the animal experiments. KAE and DJ conceived of the study, participated in its design and coordination and helped to draft the manuscript. All authors read and approved the final manuscript.

\section{Acknowledgments}

The skillfull technical assistance of Sylvia Trinh, Ludmilla Frick, Gisela Becker, Alexandra Tietz, Angela Funk and Andreas Griesbach is gratefully acknowledged.

\section{Funding sources}

This study was financially supported by the Deutsche Krebshilfe and the Dietmar Hopp Stiftung, Germany. The study sponsors were not involved in the data analysis, interpretation, writing and submission of the manuscript.

\begin{abstract}
Author details
${ }^{1}$ Department of Radiation Oncology, University of Heidelberg, Heidelberg, Germany. '2Department of Pediatric Oncology, Hematology and Immunology, University Children's, Hospital of Heidelberg, Heidelberg, Germany. ${ }^{3}$ Institute of Pathology, University of Heidelberg, Heidelberg, Germany. ${ }^{4}$ Department of Radiation Oncology, German Cancer Research Center, Heidelberg, Germany. ${ }^{5}$ German Cancer Consortium (DKTK), Heidelberg, Germany. ${ }^{6}$ National Center for Tumor Diseases (NCT), University of Heidelberg, Heidelberg, Germany.

${ }^{7}$ Pädiatrie 5, Olgahospital, Kriegsbergstr. 62, 70174 Stuttgart, Germany.
\end{abstract}

Received: 5 May 2015 Accepted: 6 July 2015

Published online: 16 July 2015

\section{References}

1. Schardt D, Elsässer T, Schulz-Ertner D. Heavy-ion tumor therapy: Physical and radio-biological benefits. Rev Mod Phys. 2010;82:383.
2. Hundsdoerfer $P$, Albrecht M, Rühl U, Fengler R, Kulozik AE, Henze G. Longterm outcome after polychemotherapy and intensive local radiation therapy of high-grade osteosarcoma. Eur J Cancer. 2009;45(14):2447-51.

3. Blattmann C, Oertel S, Schulz-Ertner D, Rieken S, Haufe S, Ewerbeck V, et al. Non-randomized therapy trial to determine the safety and efficacy of heavy ion therapy in patients with non-resectable osteosarcomas. BMC Cancer. 2010;10:96.

4. Sugahara S, Kamada T, Imai R, Tsuji H, Kameda N, Okada T, et al. Carbon ion radiotherapy for localized primary sarcoma of the extremities: results of a phase I/II trial. Working group for the bone and soft tissue sarcomas. Radiother Oncol. 2012;105(2):226-31.

5. Matsunobu A, Imai R, Kamada T, Imaizumi T, Tsuji H, Tsujii H, et al. Impact of carbon ion radiotherapy for unresectable osteosarcoma of the trunk Working group for bone and soft tissue sarcomas. Cancer. 2012;118(18):4555-63.

6. Combs SE, Kessel KA, Herfarth K, Jensen A, Oertel S, Blattmann C, et al. Treatment of pediatric patients and young adults with particle therapy at the Heidelberg lon Therapy Center (HIT): establishment of workflow and initial clinical data. Radiat Oncol. 2012:7:170.

7. Perego P, Zuco V, Gatti L, Zunino F. Sensitization of tumor cells by targeting histone deacetylases. Biochem Pharmacol. 2012;83(8):987-94.

8. Entin-Meer M, Yang X, Vandenburg SR, Lamborn KR, Nudelman A, Rephaeli A, et al. In vivo efficacy of a novel histone deacetylase inhibitor in combination with radiation for the treatment of gliomas. Neuro Oncol. 2007;9:82-8.

9. Folkvord S, Ree AH, Furre T, Halvorsen T, Flatmark K. Radiosensitization by SAHA in experimental colorectal carcinoma models - in vivo effects and relevance of histone acetylation status. Int J Radiat Oncol Biol Phys. 2009;74:546-52.

10. Munshi A, Kurland JF, Nishikawa T, Tanaka T, Hobbs ML, Tucker SL, et al. Histone deacetylase inhibitors radiosensitize human melanoma by suppressing DNA repair activity. Clin Cancer Res. 2005;11:4912-22.

11. Thiemann M, Oertel S, Ehemann V, Weichert W, Stenzinger A, Bischof M, et al. In vivo efficacy of the histone deacetylase inhibitor suberoylanilide hydroxamic acid in combination with radiotherapy in a malignant rhabdoid tumor mouse model. Radiat Oncol. 2012;7:52.

12. Slingerland M, Guchelaar HJ, Gelderblom H. Histone deacetylase inhibitors: an overview of the clinical studies in solid tumors. Anticancer Drugs. 2014;25(2):140-9.

13. Blattmann C, Thiemann M, Stenzinger A, Christmann A, Roth E, Ehemann V, et al. Radiosensitization by Histone Deacetylase inhibition in an Osteosarcoma mouse model. Strahlenther Onkol. 2013;189(11):957-66.

14. Blattmann C, Oertel S, Ehemann V, Thiemann M, Huber PE, Bischof M, et al. Enhancement of radiation response in osteosarcoma and rhabdomyosarcoma cell lines by histone deacetylase inhibition. Int J Radiat Oncol Biol Phys. 2010;78:237-45.

15. Oertel S, Thiemann M, Richter K, Weber KJ, Huber PE, Perez RL, et al. Combination of suberoylanilide hydroxamic acid with heavy ion therapy shows promising effects in infantile sacroma cell lines. Radiat Oncol. 2011;6:119.

16. Battmann C, Thiemann T, Stenzinger A, Weichert W, Bischof M, Lopez Perez $\mathrm{R}$, et al. Radiosensitization by Histone Deacetylase Inhibition in an Osteosarcoma mouse model. Strahlenther Onkol. 2013;189(11):957-66.

17. Bielack SS, Carrle D, Hardes J, Schuck A, Paulussen M. Bone tumors in adolescents and young adults. Curr Treat Options Oncol. 2008;9:67-80.

18. Bielack SS, Kempf-Bielack B, Delling G, Exner GU, Flege S, Helmke K, et al. Prognostic factors in high-grade osteosarcoma of the extremities or trunk: an analysis of 1,702 patients treated on neoadjuvant cooperative osteosarcoma study group protocols. J Clin Oncol. 2002;20:776-90.

19. Shabason JE, Tofilon PJ, Camphausen K. Grand rounds at the national institutes of health: HDAC inhibitors as radiation modifiers, from bench to clinic. J Cell Mol Med. 2011;15:2735.

20. Bots M. R.W. Johnstone, Rational combinations using HDAC inhibitors. Clin. Cancer Res. 2009;15:3970-7.

21. Jensen $A D$, Münter MW, Debus J. Review of clinical experience with ion beam radiotherapy. Br J Radiol. 2011:84(Spec No 1):35-47.

22. Zhao $Y$, Tan J, Zhuang L, Jiang $X$, Liu ET, Yu Q. Inhibitors of histone deacetylases target the Rb-E2F1 pathway for apoptosis induction through activation of proapoptotic protein Birn. Proc Natl Acad Sci U S A. 2005:44:16090-5.

23. Lee CK, Wang S, Huang X, Ryder J, Liu B. HDAC inhibition synergistically enhances alkylator-induced DNA damage responses and apoptosis in multiple myeloma cells. Cancer Lett. 2010;296:233-40. 
24. D'Acunto CW, Fontanella B, Rodriquez M, Taddei M, Parente L, Petrella A. Histone deacetylase inhibitor FR235222 sensitizes human prostate adenocarcinoma cells to apoptosis through up-regulation of Annexin A1. Cancer Lett. 2010;295:85-91.

25. Frew AJ, Johnstone RW, Bolden JE. Enhancing the apoptotic and therapeutic effects of HDAC inhibitors. Cancer Lett. 2009;280:125-33.

26. Dickinson $M$, Johnstone RW. Histone deacetylase inhibitors: potential targets responsible for their anti-cancer effect. Invest New Drugs. 2010;28:3-20.

27. Henderson C, Mizzau M, Paroni G, Maestro R, Schneider C, Brancolini C. Role of caspases, Bid and p53 in the apoptotic response triggered by histone deacetylase inhibitors trichostatin-A (TSA) and suberoylanilide hydroxamic acid (SAHA). J Biol Chem. 2003;278:12579-89.

28. Johnstone RW, Licht JD. Histone seacetylase inhibitors in cancer therapy: Is transcription the primary target? Cancer Cell. 2003;4:13-8.

29. Vousden $\mathrm{KH}$, Lu X. Live or let die: the cell's response to p53. Nat Rev Cancer. 2002;2:594-604.

30. Zhao Y, Lu S, Wu L, Chai G, Wang H, Chen Y, et al. Acetylation of p53 at lysine $373 / 382$ by the histone deacetylase inhibitor depsipeptide induces expression of p21(Waf1/Cip1). Mol Cell Biol. 2006;26:2782-90.

31. Kawano T, Akiyama M, Agawa-Ohta M, Mikami-Terao Y, Iwase S, Yanagisawa $T$, et al. Histone deacetylase inhibitors valproic acid and depsipeptide sensitize retinoblastoma cells to radiotherapy by increasing $\mathrm{H} 2 \mathrm{AX}$ phosphorylation and p53 acetylation-phosphorylation. Int J Oncol. 2010;37:787-95.

32. Bridges KA, Hirai H, Buser CA, Brooks C, Liu H, Buchholz TA, et al. MK-1775, a novel wee1 kinase inhibitor radiosensitizes p53-defective human tumor cells. Clin Cancer Res. 2011;17:5638-48.

33. Bolden JE, Peart MJ, Johnstone RW. Anticancer activities of histone deacetylase inhibitors. Nat Rev. 2006:5:769-84.

34. Mottet D, Castronovo V. Histone deacetylases: anti-angiogenic targets in cancer therapy. Curr Cancer Drug Targets. 2010;10(8):898-913.

\section{Submit your next manuscript to BioMed Central and take full advantage of:}

- Convenient online submission

- Thorough peer review

- No space constraints or color figure charges

- Immediate publication on acceptance

- Inclusion in PubMed, CAS, Scopus and Google Scholar

- Research which is freely available for redistribution 